\title{
Sevoflurane inhibits the migration and invasion of glioma cells by upregulating microRNA-637
}

\author{
WENBO YI $^{1 *}$, DONGLIANG LI ${ }^{1 *}$, YONGMIN GUO ${ }^{1}$, YAN ZHANG ${ }^{1}$, BIN HUANG $^{2}$ and XINGANG $\mathrm{LI}^{2}$ \\ ${ }^{1}$ Department of Anesthesiology, Qilu Hospital, Shandong University; ${ }^{2}$ Department of Neurosurgery, Qilu Hospital of \\ Shandong University and Brain Science Research Institute, Shandong University, Jinan, Shandong 250012, P.R. China
}

Received November 4, 2015; Accepted November 3, 2016

DOI: 10.3892/ijmm.2016.2797

\begin{abstract}
Cancer cell migration and invasion are essential features of the metastatic process. Volatile anesthetic sevoflurane inhibits the migration and invasion of multiple cancer cell lines; however, its effects on glioma cells are unclear. Emerging evidence suggests that microRNA (miRNA)-637 regulates glioma cell migration and invasion through the Aktl pathway. Sevoflurane has been shown to modulate a number of miRNAs. In the present study, we examined whether sevoflurane inhibits glioma cell migration and invasion and, if so, whether these beneficial effects are mediated by miRNA-637. U251 glioma cells were treated without (control) or with sevoflurane at low, moderate or high concentrations for $6 \mathrm{~h}$. To explore the molecular mechanisms, an additional group of U251 cells was treated with a miRNA-637 inhibitor prior to treatment with a high concentration of sevoflurane. Compared with the control group, sevoflurane inhibited the migration and invasion of U251 cells in a dose-dependent manner. Molecular analyses revealed that sevoflurane increased the expression of miRNA-637 and decreased the expression of Akt1 and phosphorylated Akt1 in a dose-dependent manner. Moreover, the inhibitory effects of sevoflurane on U251 cell migration and invasion were completely abolished by pre-treatment with miRNA-637 inhibitor, which reversed the sevoflurane-induced reduction in the expression of Akt1 and phosphorylated Akt1 in the U251 cells. These results demonstrate that sevoflurane inhibits glioma cell migration and invasion and that these beneficial effects are mediated by the upregulation of miRNA-637, which suppresses Akt1 expression and activity. These findings may have signifi-
\end{abstract}

Correspondence to: Dr Xingang Li, Department of Neurosurgery, Qilu Hospital of Shandong University and Brain Science Research Institute, Shandong University, 44 Wenhua Xi Road, Jinan, Shandong 250012, P.R. China

E-mail: lixg@sdu.edu.cn

*Contributed equally

Key words: volatile anesthetics, gliomas, migration and invasion, microRNA-637, Akt1 cant clinical implications for anesthesiologists regarding the choice of volatile anesthetic agents for the surgical resection of gliomas to prevent metastases and improve patient outcomes.

\section{Introduction}

Malignant glioma is the most common subtype of primary brain tumor, and is characterized by rapid progression, high resistance to traditional and newer targeted therapeutic approaches and a poor clinical outcome (1-4). Surgical resection is the primary treatment for patients suffering from glioma. However, surgery may also increase the likelihood of cancer dissemination and metastasis due to the release of tumor cells into the circulation at the time of surgery. Therefore, the prevention of tumor cell dissemination during surgery is an important strategy with which to reduce cancer recurrence and improve the overall survival following tumor resection.

Tumor cell migration and invasion are considered to be primary features of the metastatic process (5). Anesthetics and anesthesia techniques have been shown to have an impact on tumor cell migration and invasion that can possibly influence the long-term outcome in patients undergoing cancer surgery (6). Thus, it is important to select appropriate anesthetics and anesthesia techniques that have inhibitory properties against the migration and invasion of tumor cells in order to eliminate the risk of tumor cell dissemination during the surgical removal of tumors. Sevoflurane, a volatile anesthetic agent, is widely used in neurosurgery. It has been demonstrated that sevoflurane exerts anti-proliferative effects on SW620 colon cancer cells and in Caco-2 laryngeal cancer cells $(7,8)$, and prevents the migration and invasion of lung cancer cells (9). However, the effects of sevoflurane on the migration and invasion of glioma cells are unclear.

MicroRNAs (miRNAs or miRs) are small non-coding RNA molecules (approximately 21-25 nucleotides in length) that play important roles in virtually all biological pathways in mammals and other multicellular organisms (10). miRNAs have been shown to influence numerous cancer-relevant processes, such as proliferation, cell cycle control, apoptosis, differentiation, migration and metabolism (10-12). A recent in vivo and in vitro study demonstrated that the expression of miRNA-637 was significantly lower in clinical glioma tissues than in normal brain tissues, whereas the introduction of miRNA-637 prevented glioma cell growth, migration and inva- 
sion (13). Sevoflurane has been reported to modulate multiple miRNAs in the brain and in peripheral tissues (14-16).

In the present study, we examined whether sevoflurane inhibits glioma cell migration and invasion and, if so, whether these beneficial effects are mediated by miRNA-637.

\section{Materials and methods}

Cell line and cell culture. The human glioma cell line, U251, was obtained from the Chinese Academy of Sciences (Shanghai, China) and maintained in Dulbecco's modified Eagle's medium (DMEM) containing 10\% fetal calf serum (both Hyclone, Logan, UT, USA). The cells were incubated at $37^{\circ} \mathrm{C}$ in a humidified atmosphere of $5 \% \mathrm{CO}_{2}$.

Protocol of U251 cell exposure to sevoflurane. The U251 cells were divided into 4 groups as follows: cells treated without (control, 95\% air $5 \% \mathrm{CO}_{2}$ ) or with sevoflurane (Maruishi Pharmaceutical Co., Osaka, Japan) at low (1.7\% sevoflurane mixed with $95 \%$ air $/ 5 \% \mathrm{CO}_{2}$ ), moderate $(3.4 \%$ sevoflurane mixed with $95 \%$ air $\left./ 5 \% \mathrm{CO}_{2}\right)$ and high $(5.1 \%$ sevoflurane mixed with $95 \%$ air $/ 5 \% \mathrm{CO}_{2}$ ) concentrations. To examine whether sevoflurane inhibits glioma cell migration and invasion by the modulation of miRNA-637, an additional group of U251 cells was treated with an miRNA-637 inhibitor for $48 \mathrm{~h}$ prior to exposure to a high concentration (5.1\%) of sevoflurane. The protocol for the treatment of cells with sevoflurane was in accordance with that of previous studies $(9,17)$. Briefly, the U251 cells in the exponential growth phase were seeded onto plates and incubated in a $\mathrm{CO}_{2}$ incubator (Thermo Fisher Scientific, Waltham, MA, USA) for $24 \mathrm{~h}$. The cell culture plates were then placed in an airtight glass chamber connected to an anesthesia machine Cicero-EM 8060; Drager, Lübeck, Germany). An anesthetic vaporizer (Sevorane; Abbott, Abbot Park, IL, USA) attached to the anesthesia machine was used to supply sevoflurane into the chamber. The concentrations of sevoflurane in the chamber were monitored by a gas monitor (PM 8060; Drager). After being exposed to various concentrations of sevoflurane for $6 \mathrm{~h}$, the cells were grown at $37^{\circ} \mathrm{C}$ in a $\mathrm{CO}_{2}$ incubator for an additional $24 \mathrm{~h}$ and then used for cell migration and invasion assays or molecular analyses.

Transfection of U251 cells with miRNA-637 inhibitor. The transfection of the U251 cells with miRNA-637 inhibitor was performed according to a previous study (13). siRNA targeting miR-637 (miR-637 inhibitor) was synthesized by Guangzhou RiboBio (Guangzhou, China). The sequence of the miRNA-637 inhibitor was: 5'-ACGCAGAGCCCGAAAGCCCCAGU-3'. Twelve hours prior to transfection, the cells were seeded in a 6-well plate (Nest Biotech, Shanghai, China) at 30-50\% confluence. Transfection with the siRNA was performed using TurboFect siRNA transfection reagent (Fermentas, Vilnius, Lithuania) according to the manufacturer's instructions. Fortyeight hours following transfection with siRNA, the cells were collected for further analyses.

Cell migration and invasion assays. In vitro cell migration and invasion measurements were examined as previously described (13). For the determination of cell migration, $1 \times 10^{4}$ cells in $100 \mu \mathrm{l}$ DMEM medium without fetal bovine serum were seeded on a fibronectin-coated polycarbonate membrane insert in a Transwell apparatus (Costar, Corning, NY, USA). A total of $500 \mu 1$ DMEM with $10 \%$ fetal bovine serum was added to the lower chamber as a chemoattractant. Following $6 \mathrm{~h}$ of incubation at $37^{\circ} \mathrm{C}$ in a $5 \% \mathrm{CO}_{2}$ atmosphere, the insert was washed twice with phosphate-buffered saline, and the cells on the top surface of the insert were wiped off with a cotton swab. The cells on the lower surface of the insert were fixed with methanol, stained using crystal violet solution (Sigma-Aldrich, St. Louis, MO, USA) and counted under a microscope (Nikon Eclipse TS100; Nikon, Tokyo, Japan) in 5 predetermined fields (x200 magnification). All measurements were repeated at least 3 times. The procedure for the measurement of cell invasion was similar to the measurement of cell migration, except that Matrigel (24 $\mu \mathrm{g} / \mu \mathrm{l}$; R\&D Systems, Inc., Minneapolis, MN, USA) was added to the Transwell membranes and the cells were incubated for $8 \mathrm{~h}$ at $37^{\circ} \mathrm{C}$ in a $5 \% \mathrm{CO}_{2}$ atmosphere. The cells on the lower surface were counted in the same manner as those for the determination of cell migration.

RNA isolation and real-time polymerase chain reaction (PCR). RNA was isolated from the U251 cells using TRIzol ${ }^{\circledR}$ reagent (Invitrogen, Carlsbad, CA, USA) and purification using an RNeasy mini kit (Qiagen, Valencia, CA, USA) as previously described (18). For the analysis of miRNA-637 expression, the RNA was transcribed into cDNA and amplified with specific sense primer, 5'-ACTGGGGGCTTTCGGGCTCTGCGT-3', and antisense general primer, using the miRNA PrimeScript RT Enzyme Mix kit according to the manufacturer's instructions (Takara Bio, Inc., Otsu, Japan). For the analysis of Aktl gene expression, the sense primer was 5'-CTGAGATTGTGT CAGCCCTGGA-3', and the antisense primer was 5'-CAC AGCCCGAAGTCTGTGATCTTA-3'. The expression of U6 (sense primer, 5'-CTCGCTTCGGCAGCACATATA-3') and ADP-ribosylation factor 5 (ARF5) (sense primer, 5'-ATCTG TTTCACAGTCTGGGACG-3' and antisense primer, 5'-CCTGCTTGTTGGCAAATACC-3') was also measured and used as miRNA and gene internal controls, respectively. The thermocycling conditions for PCR were as follows: $95^{\circ} \mathrm{C}$ for $10 \mathrm{~min}$ to activate DNA polymerase, followed by 45 cycles of $95^{\circ} \mathrm{C}$ for $15 \mathrm{sec}, 58^{\circ} \mathrm{C}$ (for miR-637) or $60^{\circ} \mathrm{C}$ (for Akt1) for $15 \mathrm{sec}$ and $72^{\circ} \mathrm{C}$ for $10 \mathrm{sec}$. Melting curve analysis was used to confirm the specificity of amplification. For each gene, PCR reactions were repeated 3 times.

Western blot analysis. Cell homogenates were separated by $10 \%$ sodium dodecyl sulfate-polyacrylamide gel electrophoresis (SDS-PAGE) and transferred onto a polyvinylidene fluoride membrane. The membrane was blocked in Tris-buffered saline Tween-20 (TBST) using 5\% skimmed milk and incubated overnight with rabbit polyclonal Akt (\#4691) and phosphorylated (p-) Akt (\#4058) antibodies (1:1,000; Cell Signaling Technology). Mouse monoclonal $\beta$-actin antibody (sc-47778; 1:200; Santa Cruz Biotechnology, Inc., Santa Cruz, CA, USA) was used as an internal control. The membrane was then incubated for $1 \mathrm{~h}$ with a horseradish peroxidase-conjugated anti-rabbit (sc-2030) or anti-mouse (sc-2005) immunoglobulin-G secondary antibody (1:5,000; Santa Cruz Biotechnology, Inc.) diluted with TBST. Signals were detected using an enhanced chemiluminescence (ECL) reaction system (Millipore, Billerica, MA, USA) 
A

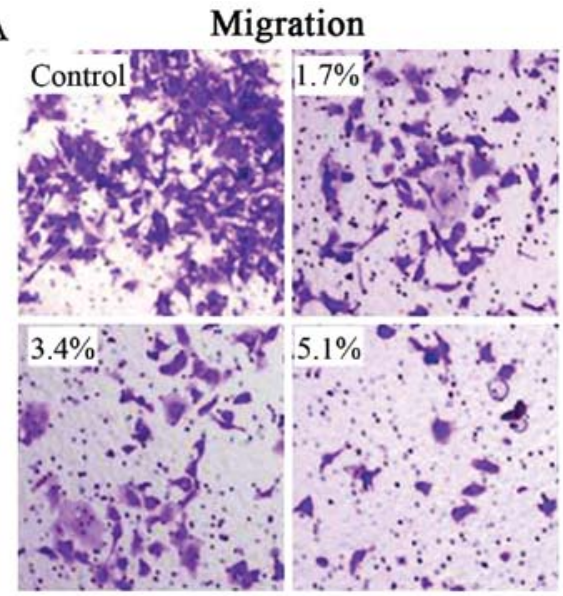

$\mathrm{C}$

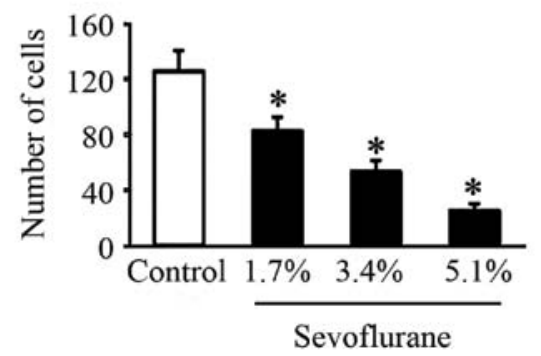

B

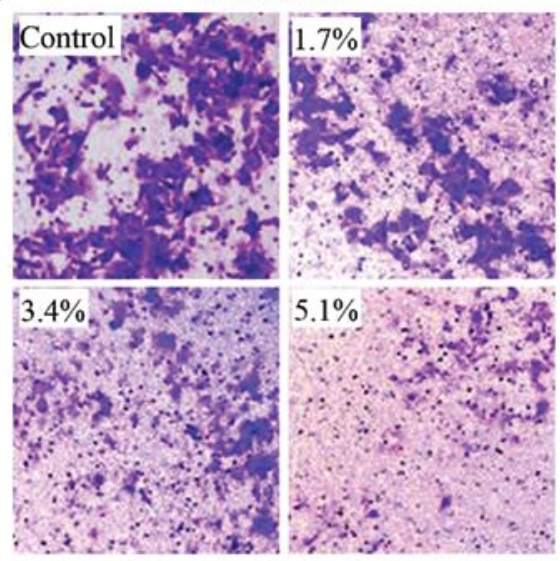

$\mathrm{D}$

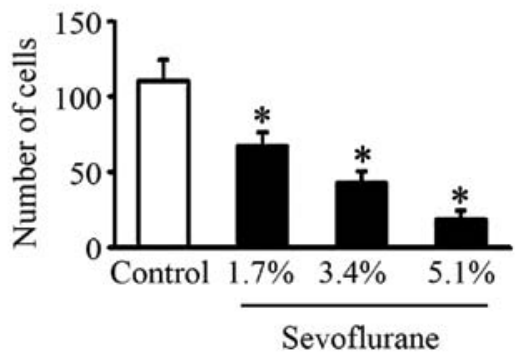

Figure 1. Effects of sevoflurane on the migration and invasion of U251 cells. The cells were treated without (control) or with sevoflurane at low (1.7\%), moderate $(3.4 \%)$ or high $(5.1 \%)$ concentrations for $6 \mathrm{~h}$. (A and B) Representative images showing the migratory and invading cells on the membranes. (C and D) Quantification of the migratory and invading cells. Data are presented as the means \pm SE from 3 independent experiments. "P $<0.05$ vs. control.

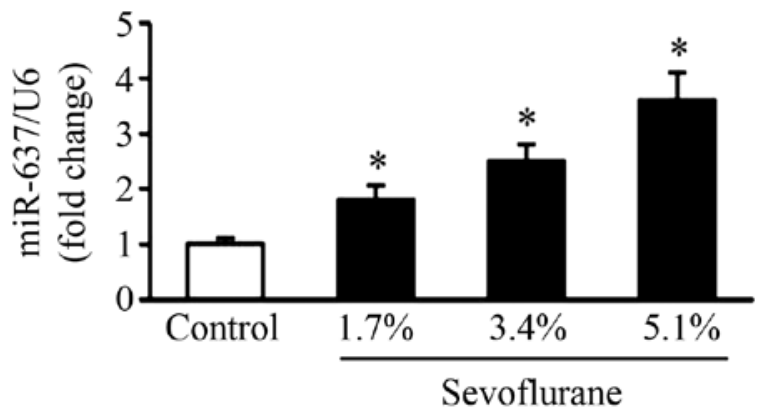

Figure 2. Effects of sevoflurane on miRNA-637 expression in U251 cells. The cells were treated without (control) or with sevoflurane at various concentrations $(1.7,3.4$ and $5.1 \%)$ for $6 \mathrm{~h}$. Data are the means $\pm \mathrm{SE}$ from 3 independent experiments and are expressed as a fold change relative to the control. ${ }^{*} \mathrm{P}<0.05$ vs. control.

and quantified using ImageJ software (National Institutes of Health, Bethesda, MD, USA).

Statistical analysis. All quantified data are presented as an average of at least triplicate samples. Values are expressed as the means \pm SE. The significance of differences in mean values was analyzed by one-way ANOVA followed by Tukey's multiple comparison tests. A value of $\mathrm{P}<0.05$ was considered to indicate a statistically significant difference.

\section{Results}

Sevoflurane inhibits the migratory and invasive abilities of glioma cells. To examine the effects of sevoflurane on glioma cell migration and invasion, the U251 cells, following treatment without or with various concentrations of sevoflurane, were cultured in a Transwell chamber. The alteration of cell migration following $6 \mathrm{~h}$ of incubation was determined. Compared with the control group, the U251 cells treated with sevoflurane exhibited a significantly decreased migratory ability in a dosedependent manner (Fig. 1A and C). For cell invasion assay, following $8 \mathrm{~h}$ of incubation, the U251 cells in all groups invaded through the matrigel. However, treatment with sevoflurane attenuated the cell invasive ability compared with the control group in a dose-dependent manner (Fig. 1B and D).

Sevoflurane induces the upregulation of miRNA-637 expression in glioma cells. To determine whether sevoflurane induces changes in miRNA-637 expression in glioma cells, we analyzed the expression level of miRNA-637 by real-time PCR following treatment of the cells with sevoflurane. The U251 cells treated with sevoflurane had significantly increased levels of miRNA-637 as compared to those of the control group, and the increases in the levels of miRNA-637 were dose-dependent (Fig. 2).

Sevoflurane suppresses Akt1 expression and activity in glioma cells. As Aktl is a crucial component of the Akt pathway involved in the promotion of cell proliferation, migration and invasion in several types of tumors (19) and is regulated by miRNA-637 (13), we then measured the expression of Akt1 and that of p-Akt1 in the U251 cells using real-time PCR and western blot analysis following treastment with sevoflurane. Compared with the control group, the U251 cells treated with sevoflurane exhibited a dose-dependent decrease in the mRNA 
A

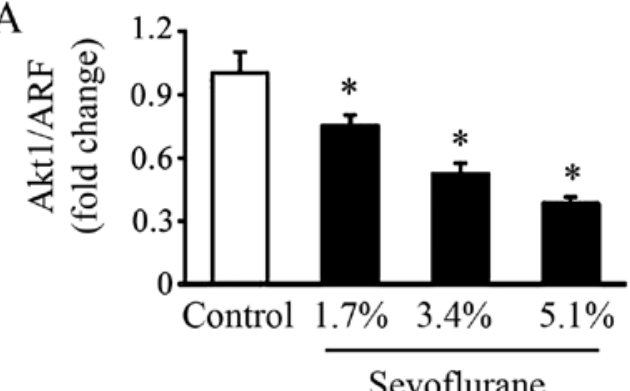

$\mathrm{C}$

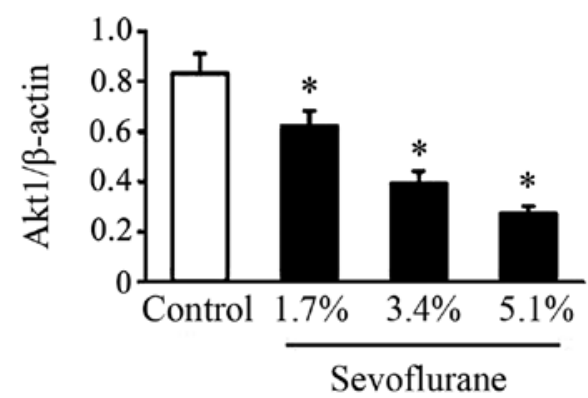

B

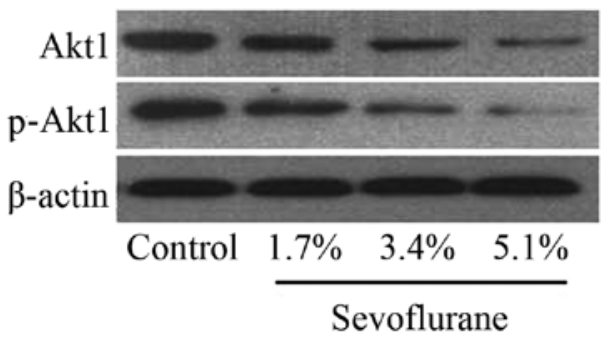

$\mathrm{D}$

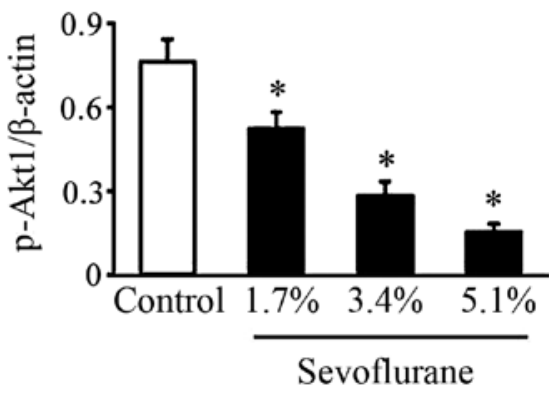

Figure 3. Effects of sevoflurane on Akt1 expression and activity. The cells were treated without (control) or with sevoflurane at various concentrations (1.7, 3.4 and $5.1 \%$ ) for 6 h. (A) mRNA expression of Akt1 measured by real-time PCR. (B-D) Protein levels of Akt1 and phosphorylated (p-)Akt1 measured by western blot analysis. Data are presented as the means \pm SE from 3 independent experiments. ${ }^{*} \mathrm{P}<0.05$ vs. control.

expression of Akt1 (Fig. 3A) and in the protein levels of Akt1 and p-Akt1 (Fig. 3B-D). Thus, the expression of Akt1 and p-Akt1 negatively correlate with the expression of miRNA-637 in glioma cells.

The sevoflurane-induced inhibition of the migration and invasion of glioma cells is mediated by miRNA-637. To further confirm whether the inhibitory effects of sevoflurane on glioma cell migration and invasion are mediated by miRNA-637, we treated the U251 cells with a miRNA-637 inhibitor prior to exposure to a high concentration of sevoflurane. We found that pre-treatment of the U251 cells with miRNA-637 inhibitor completely abolished the inhibitory effects of sevoflurane on U251 cell migration and invasion (Fig. 4).

The sevoflurane-induced upregulation of miRNA-637 inhibits glioma cell migration and invasion via the suppression of Aktl. To confirm that the sevoflurane-induced upregulation of miRNA-637 inhibits glioma cell migration and invasion via the suppression of Akt1, we measured the expression of Akt1 and p-Akt1 in U251 cells pre-treated with miRNA-637 inhibitor followed by exposure to a high concentration of sevoflurane. Our results revealed that the decreases in the mRNA expression of Akt1 and protein levels of Akt1 and p-Akt1 in the U251 cells induced by sevoflurane were reversed by pre-treatment with miRNA-637 inhibitor (Fig. 5).

\section{Discussion}

The novel findings of this study are the following: i) sevoflurane inhibits the migratory and invasive abilities of glioma cells; ii) the inhibitory effects of sevoflurane on glioma cell migration and invasion are mediated by the upregulation of miRNA-637, which suppresses Akt1 expression and activity. To the best of our knowledge, this is the first study to demonstrate the inhibitory effects of sevoflurane on glioma cell migration and invasion, and to demonstrate the possible underlying mechanisms.

Metastasis, the leading cause for the resultant mortality of patients with cancer, is receiving increasing attention in both scientific and clinical research. Metastasis is an exceedingly complex process, which includes the detachment of the tumor cells from the primary site, the degradation of the extracellular matrix and the penetration of the tumor cells into the blood vessel walls (20). All of these processes are associated with the invasive and migration properties of tumor cells (20). It has been reported that surgical procedures may induce the release of tumor cells into the circulation, resulting in the invasive and migratory potential of tumor cells (21), and promoting the ability of tumor metastasis (22). Thus, preventing the metastatic potential of tumor cells during surgery to remove the tumor is a challenging topic. The volatile anesthetic, sevoflurane, has been shown to attenuate the proliferation of SW620 colon cancer cells and Caco-2 laryngeal cancer cells $(7,8)$, and to inhibit the migration and invasion of lung cancer cells (9). The present study extended these findings by showing that sevoflurane significantly prevented the migration and invasion of glioma cells.

Emerging evidence has suggested that alterations of a group of miRNAs are involved in the pathogenesis of many types of cancer, including glioma (23-26). Several deregulated miRNAs target key gene products to regulate cell proliferation, invasion and migration (27-29). The deregulation of miRNA-637, which functions as a tumor suppressor, has been shown to be associated with the initiation and progression of several types of human cancers, such as hepatocellular carcinoma [Zhang et al (30)], breast cancer (31) and follicular thyroid carcinoma (32). A recent study demonstrated that the expression level of miRNA-637 
A

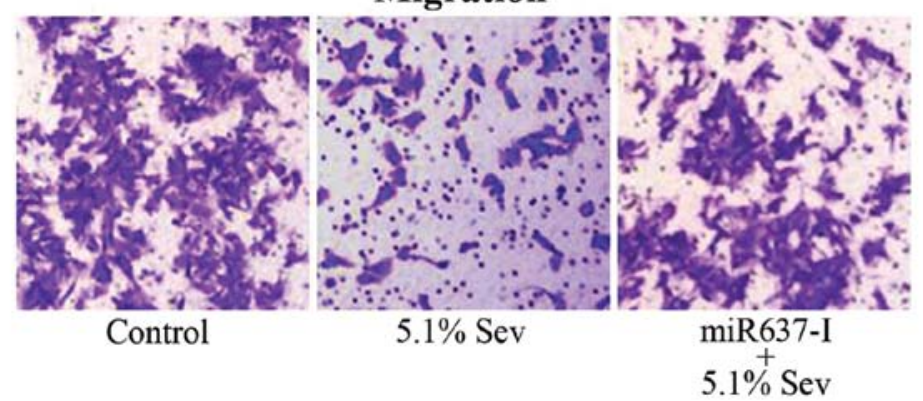

$\mathrm{C}$
B

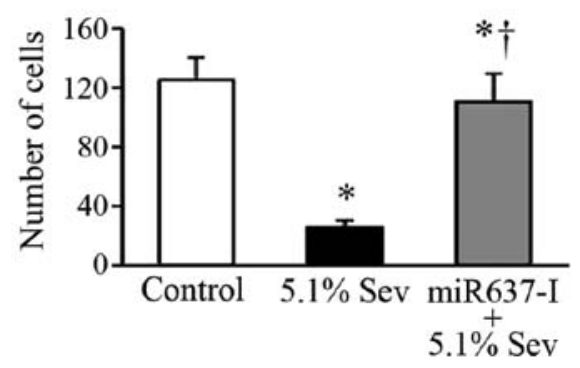

D

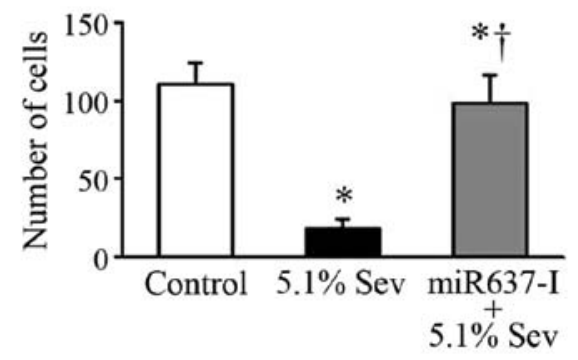

Figure 4. Effects of miRNA-637 inhibitor (miR637-I; transfection of cells with siRNA targeting miRNA-637) on the migration and invasion of U251 cells treated with sevoflurane. The miRNA-637 inhibitor completely abolished the inhibitory effects of sevoflurane on U251 cell migration and invasion. The cells were treated without or with a miRNA-637 inhibitor prior to treatment with 5.1\% sevoflurane. Cells without any treatment served as the controls. (A and C) Representative images showing the migratory and invading cells on the membranes. (B and D) Quantification of the migratory and invading cells. Data are presented as the means \pm SE from 3 independent experiments. ${ }^{*} \mathrm{P}<0.05$ vs. control (without treatment); ${ }^{\dagger} \mathrm{P}<0.05$ vs. cells treated with $5.1 \%$ sevoflurane alone. Sev, sevoflurane.

A

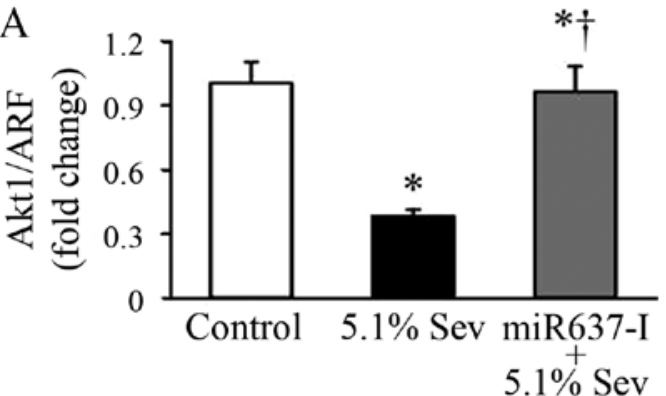

$\mathrm{C}$

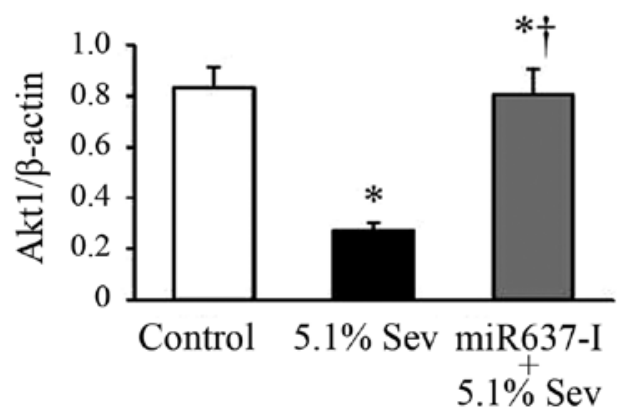

B

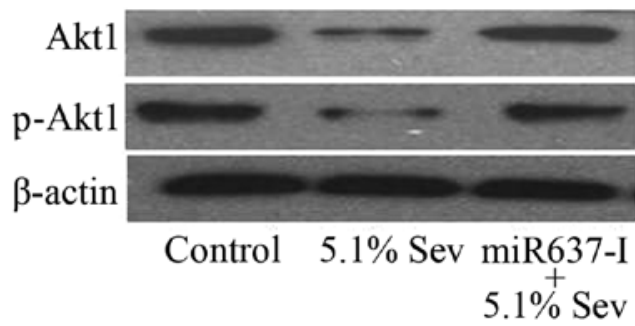

D

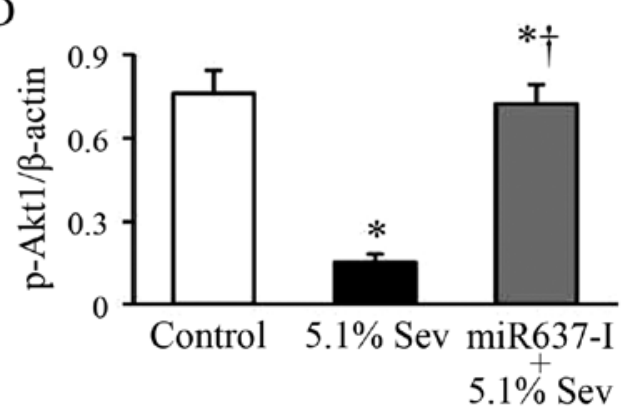

Figure 5. Effects of miRNA-637 inhibitor (miR637-I; transfection of cells with siRNA against miRNA-637) on Akt1 expression in U251 cells. miRNA-637 inhibitor (miR637-I) reversed the sevoflurane-induced reduction in the expression of Akt1 and phosphorylated (p-)Akt1 in U251 cells. The cells were treated without or with a miRNA-637 inhibitor prior to treatment with $5.1 \%$ sevoflurane. Cells without any treatment served as the controls. (A) mRNA expression of Akt1 measured by real-time PCR. (B-D) Protein levels of Akt1 and phosphorylated Akt1 measured by western blot analysis. Data are presented as the means $\pm \mathrm{SE}$ from 3 independent experiments. ${ }^{*} \mathrm{P}<0.05$ vs. control (without treatment); ${ }^{\dagger} \mathrm{P}<0.05$ vs. cells treated with $5.1 \%$ sevoflurane alone. Sev, sevoflurane.

was significantly reduced in clinical glioma tissues compared with normal brain tissues (13); moreover, the overexpression of
miRNA-637 markedly suppressed glioma cell growth, migration and invasion in vitro and in vivo, whereas the inhibition 


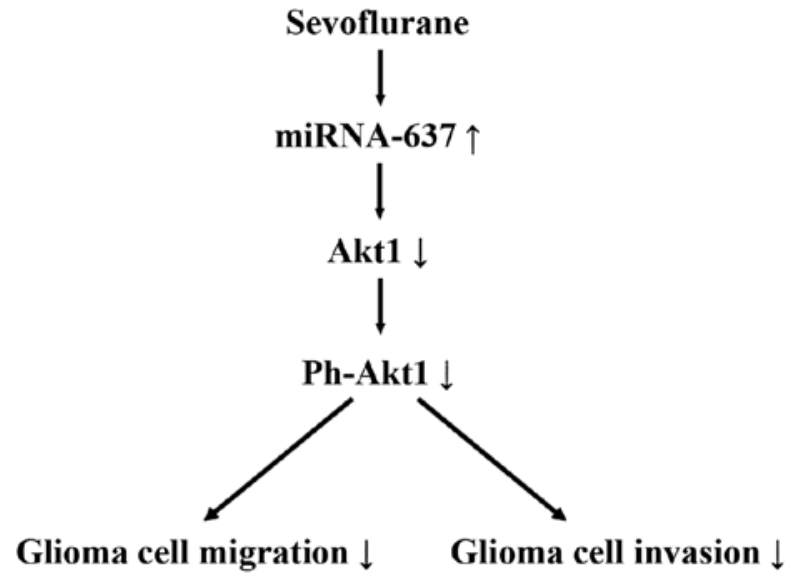

Figure 6. Schematic representation of the inhibition of the migration and invasion of glioma cells by sevoflurane. Sevoflurane upregulates the expression of miRNA-637, which suppresses the expression and activity of Akt1, and prevents glioma cell migration and invasion.

of miRNA-637 resulted in a significant increase in glioma cell invasion and migration (13). These results suggest that miRNA-637 exerts significant inhibitory effects on the migration, invasion and tumorigenesis of glioma cells. A number of anesthetics, including sevoflurane, have been reported to cause alterations in the expression of many miRNAs in both brain and peripheral tissues (14-16,33). In the present study, we found that the inhibitory effects of sevoflurane on glioma cell migration and invasion were dose-dependently associated with an increase in the expression of miRNA-637. Importantly, the inhibition of miRNA-637 with an inhibitor (with siRNA transfection) completely abolished the inhibitory effects of sevoflurane on glioma cell migration and invasion. These results clearly indicate that sevoflurane prevents the migration and invasion of glioma cells by upregulating miRNA- 637 .

Akt1 is an essential component of the Akt pathway that regulates cell proliferation, migration and invasion in several types of tumors (19). The silencing of Akt1 inhibits the growth and invasion of glioma cells by decreasing phosphorylated Akt, $\beta$-catenin, phosphorylated Foxo1 and cyclin D1 and inducing the expression of Foxo1, whereas an increase in Akt1 protein levels is associated with the enhanced migration and invasion of glioma cells $(13,34,35)$. Akt1 is a direct target gene of miRNA-637 (13). The overexpression of miRNA-637 has been shown to prevent the proliferation, migration and invasion of glioma cells through the direct targeting of Akt1 (13). Our results revealed that the upregulation of miRNA-637 in glioma cells by sevoflurane suppressed both the Akt1 mRNA and protein levels in a dose-dependent manner, and subsequently inhibited the p-Akt1 levels. Moreover, the inhibition of miRNA-637 with an inhibitor (by siRNA transfection) completely reversed the sevoflurane-induced decrease in the expression of Akt1 and p-Akt1. These findings suggest that the upregulation of miRNA-637 by sevoflurane inhibits glioma cell migration and invasion by suppressing Akt1 expression and activity.

In conclusion, the present study demonstrates that the volatile anesthetic, sevoflurane, inhibits glioma cell migration and invasion, and that these beneficial effects are mediated by the upregulation of miRNA-637, which suppresses Akt1 expres- sion and activity (Fig. 6). These findings may have significant clinical implications for anesthesiologists regarding the choice of volatile anesthetic agents for the surgical resection of gliomas in order to prevent metastases and improve patient outcomes.

\section{Acknowledgements}

The present study was supported by the National Natural Science Foundation of China (grant nos. 81572487 and 81172404 to X. Li), the Major Project of Science and Technology of Shandong Province (grant no. 2016GSF201070 to D. Li) and the Shandong Provincial Outstanding Medical Academic Professional Program and Special foundation for Taishan Scholars (no. ts20110814).

\section{References}

1. Maher EA, Furnari FB, Bachoo RM, Rowitch DH, Louis DN, Cavenee WK and DePinho RA: Malignant glioma: Genetics and biology of a grave matter. Genes Dev 15: 1311-1333, 2001.

2. Emdad L, Dent P, Sarkar D and Fisher PB: Future approaches for the therapy of malignant glioma: Targeting genes mediating invasion. Future Oncol 8: 343-346, 2012.

3. Stupp R, Hegi ME, Mason WP, van den Bent MJ, Taphoorn MJ, Janzer RC, Ludwin SK, Allgeier A, Fisher B, Belanger K, et al; European Organisation for Research and Treatment of Cancer Brain Tumour and Radiation Oncology Groups; National Cancer Institute of Canada Clinical Trials Group: Effects of radiotherapy with concomitant and adjuvant temozolomide versus radiotherapy alone on survival in glioblastoma in a randomised phase III study: 5-year analysis of the EORTC-NCIC trial. Lancet Oncol 10: 459-466, 2009.

4. Lefranc F, Brotchi J and Kiss R: Possible future issues in the treatment of glioblastomas: Special emphasis on cell migration and the resistance of migrating glioblastoma cells to apoptosis. J Clin Oncol 23: 2411-2422, 2005.

5. Friedl $\mathrm{P}$ and Wolf $\mathrm{K}$ : Tumour-cell invasion and migration: Diversity and escape mechanisms. Nat Rev Cancer 3: 362-374, 2003.

6. Snyder GL and Greenberg S: Effect of anaesthetic technique and other perioperative factors on cancer recurrence. Br J Anaesth 105: 106-115, 2010.

7. Kvolik S, Glavas-Obrovac L, Bares V and Karner I: Effects of inhalation anesthetics halothane, sevoflurane, and isoflurane on human cell lines. Life Sci 77: 2369-2383, 2005

8. Kvolik S, Dobrosevic B, Marczi S, Prlic L and Glavas-Obrovac L: Different apoptosis ratios and gene expressions in two human cell lines after sevoflurane anaesthesia. Acta Anaesthesiol Scand 53: 1192-1199, 2009.

9. Liang $\mathrm{H}$, Gu M, Yang $\mathrm{C}$, Wang $\mathrm{H}$, Wen $\mathrm{X}$ and Zhou $\mathrm{Q}$ : Sevoflurane inhibits invasion and migration of lung cancer cells by inactivating the p38 MAPK signaling pathway. J Anesth 26: 381-392, 2012.

10. Jansson MD and Lund AH: MicroRNA and cancer. Mol Oncol 6: 590-610, 2012.

11. Liu Y, Zhou Y, Feng X, An P, Quan X, Wang H, Ye S, Yu C, He Y and Luo H: MicroRNA-126 functions as a tumor suppressor in colorectal cancer cells by targeting CXCR4 via the AKT and ERK1/2 signaling pathways. Int J Oncol 44: 203-210, 2014.

12. Gu JJ, Gao GZ and Zhang SM: miR-218 inhibits the migration and invasion of glioma U87 cells through the Slit2-Robo1 pathway. Oncol Lett 9: 1561-1566, 2015.

13. Que T, Song Y, Liu Z, Zheng S, Long H, Li Z, Liu Y, Wang G, Liu Y, Zhou J, et al: Decreased miRNA-637 is an unfavorable prognosis marker and promotes glioma cell growth, migration and invasion via direct targeting Akt1. Oncogene 34: 4952-4963, 2015.

14. Takeuchi J, Sakamoto A and Takizawa T: Sevoflurane anesthesia persistently downregulates muscle-specific microRNAs in rat plasma. Int J Mol Med 34: 291-298, 2014.

15. Goto G, Hori Y, Ishikawa M, Tanaka S and Sakamoto A: Changes in the gene expression levels of microRNAs in the rat hippocampus by sevoflurane and propofol anesthesia. Mol Med Rep 9: 1715-1722, 2014. 
16. Otsuki T, Ishikawa M, Hori Y, Goto G and Sakamoto A: Volatile anesthetic sevoflurane ameliorates endotoxin-induced acute lung injury via microRNA modulation in rats. Biomed Rep 3: 408-412, 2015.

17. Roesslein M1, Frick M, Auwaerter V, Humar M, Goebel U, Schwer C, Geiger KK, Pahl HL, Pannen BH and Loop T: Sevoflurane-mediated activation of p38-mitogen-activated stresskinase is independent of apoptosis in Jurkat T-cells. Anesth Analg 106: 1150-1160, 2008.

18. Li D, Wang C, Li N and Zhang L: Propofol selectively inhibits nuclear factor- $\mathrm{\kappa B}$ activity by suppressing p38 mitogen-activated protein kinase signaling in human EA.hy926 endothelial cells during intermittent hypoxia/reoxygenation. Mol Med Rep 9: 1460-1466, 2014.

19. Li GQ, Zhang Y, Liu D, Qian YY, Zhang H, Guo SY, Sunagawa M, Hisamitsu T and Liu YQ: PI3 kinase/Akt/HIF-1 $\alpha$ pathway is associated with hypoxia-induced epithelial-mesenchymal transition in fibroblast-like synoviocytes of rheumatoid arthritis. Mol Cell Biochem 372: 221-231, 2013.

20. Bozzuto G, Ruggieri P and Molinari A: Molecular aspects of tumor cell migration and invasion. Ann Ist Super Sanita 46: 66-80, 2010.

21. Coffey JC, Wang JH, Smith MJ, Bouchier-Hayes D, Cotter TG and Redmond HP: Excisional surgery for cancer cure: Therapy at a cost. Lancet Oncol 4: 760-768, 2003.

22. Yamaguchi K, Takagi Y, Aoki S, Futamura M and Saji S: Significant detection of circulating cancer cells in the blood by reverse transcriptase-polymerase chain reaction during colorectal cancer resection. Ann Surg 232: 58-65, 2000.

23. Hobert O: miRNAs play a tune. Cell 131: 22-24, 2007.

24. Bartel DP: MicroRNAs: Target recognition and regulatory functions. Cell 136: 215-233, 2009.

25. Yin D, Ogawa S, Kawamata N, Leiter A, Ham M, Li D, Doan NB, Said JW, Black KL and Phillip Koeffler H: miR-34a functions as a tumor suppressor modulating EGFR in glioblastoma multiforme. Oncogene 32: 1155-1163, 2013.

26. Chen Z, Li D, Cheng Q, Ma Z, Jiang B, Peng R, Chen R, Cao Y and Wan X: MicroRNA-203 inhibits the proliferation and invasion of U251 glioblastoma cells by directly targeting PLD2. Mol Med Rep 9: 503-508, 2014.
27. Fang L, Deng Z, Shatseva T, Yang J, Peng C, Du WW, Yee AJ, Ang LC, He C, Shan SW and Yang BB: MicroRNA miR-93 promotes tumor growth and angiogenesis by targeting integrin- $\beta 8$. Oncogene 30: 806-821, 2011.

28. Dontula R, Dinasarapu A, Chetty C, Pannuru P, Herbert E, Ozer H and Lakka SS: MicroRNA 203 modulates glioma cell migration via Robo1/ERK/MMP-9 signaling. Genes Cancer 4: 285-296, 2013.

29. Wu S, Lin Y, Xu D, Chen J, Shu M, Zhou Y, Zhu W, Su X, Zhou Y, Qiu P, et al: MiR-135a functions as a selective killer of malignant glioma. Oncogene 31: 3866-3874, 2012.

30. Zhang JF, He ML, Fu WM, Wang H, Chen LZ, Zhu X, Chen Y, Xie D, Lai P, Chen G, et al: Primate-specific microRNA-637 inhibits tumorigenesis in hepatocellular carcinoma by disrupting signal transducer and activator of transcription 3 signaling. Hepatology 54: 2137-2148, 2011.

31. Leivonen SK, Sahlberg KK, Mäkelä R, Due EU, Kallioniemi O, Børresen-Dale AL and Perälä M: High-throughput screens identify microRNAs essential for HER2 positive breast cancer cell growth. Mol Oncol 8: 93-104, 2014.

32. Stokowy T, Wojtaś B, Fujarewicz K, Jarząb B, Eszlinger M and Paschke R: miRNAs with the potential to distinguish follicular thyroid carcinomas from benign follicular thyroid tumors: Results of a meta-analysis. Horm Metab Res 46: 171-180, 2014.

33. Ishikawa M, Tanaka S, Arai M, Genda Y and Sakamoto A: Differences in microRNA changes of healthy rat liver between sevoflurane and propofol anesthesia. Anesthesiology 117: 1245-1252, 2012.

34. Włodarski P, Grajkowska W, Łojek M, Rainko K and Jóźwiak J: Activation of Akt and Erk pathways in medulloblastoma. Folia Neuropathol 44: 214-220, 2006.

35. Schlegel J, Piontek G, Budde B, Neff F and Kraus A: The Akt/protein kinase B-dependent anti-apoptotic pathway and the mitogen-activated protein kinase cascade are alternatively activated in human glioblastoma multiforme. Cancer Lett 158 : 103-108, 2000. 